\title{
Dissociated centriole development as a cause of spermatid malformation in man
}

\author{
A. F. Holstein, W. B. Schill* and H. Breucker \\ Department of Microscopical Anatomy, Anatomical Institute, University of Hamburg, \\ Martinistrasse 52, D-2000 Hamburg 20; and *Department of Dermatology, Andrology Unit, \\ Ludwig-Maximilians-University, D-8000 Munich 2, Federal Republic of Germany
}

\begin{abstract}
Summary. Ultrastructural study of a testicular biopsy from an infertile man with decapitated spermatozoa revealed a hitherto undescribed type of malformation. It was caused by a dissociation between the proximal and distal centrioles during the first steps of spermatid differentiation. The disconnection probably occurred because of the lack of striated columns in the connecting piece. Up to $40 \%$ of the separately developed and released tails showed a normal motility in the ejaculate.
\end{abstract}

\section{Introduction}

Defects of the tail are frequent anomalies occurring during spermatid differentiation (Holstein, 1975 ) and relate to the structures of the middle as well as of the principal piece. Also, implantation of the flagellum at the spermatid nucleus may fail to appear. However, anomalies of the centrioles, normally lying in T-formation, are seldom observed; it is mostly their associated structures, e.g. the spindle-shaped body or the centriolar adjunct, that are affected. A general omission or disappearance of the $\mathrm{T}$-formation of the centrioles during spermatid differentiation has not so far been observed; it is a rare type of malformation which only occasionally has been found as a singular observation amongst other defects. A failure of implantation of the flagellum as a whole as well as the dissociation of the centrioles both result in decapitated spermatozoa in the ejaculate, as described for the human by Perotti et al. (1981) and Baccetti et al. (1984).

In the testicular biopsy of an infertile patient with decapitated spermatozoa in the ejaculate a high percentage of the mature spermatids had centrioles that were not arranged in T-formation. The aim of this investigation was to study the morphogenesis of this malformation of spermatid differentiation.

\section{Materials and Methods}

The patient was a 25 -year-old man who underwent a testicular biopsy because of a teratozoospermia previously diagnosed by light microscopy (Faidt, 1979). Several ejaculates obtained at different times before this operation were used for standard andrological investigations, for histologically stained smears and for electron microscopy. The testicular tissue of the biopsy was fixed in phosphate-buffered $6 \%$ glutaraldehyde, postfixed in phosphate-buffered $1 \% \mathrm{OsO}_{4}$, and after dehydration embedded in Epon 812 . Ultrathin sections, contrasted with lead citrate and uranyl acetate, were studied in a Philips EM 300 transmission electron microscope. 
Results

The examination of the spermiogram showed values in the standard range, with a volume of $2.6 \mathrm{ml}$, pH 6.7, sperm density of about $66 \times 10^{6} / \mathrm{ml}$, motility of the spermatozoa (normal and decapitated) of $40 \%$, normal viscosity of the ejaculate (liquefaction $20 \mathrm{~min}$ after ejaculation) and the fructose content $(2 \cdot 20 \mathrm{mg} / \mathrm{ml})$. However, light microscopic examination of the smears revealed a very high degree of pathological sperm forms. Only about $5 \%$ of the spermatozoa in the ejaculate appeared to be normal while the remaining $95 \%$ consisted predominantly of tails bearing an eosinophilic knob instead of a normal head with nucleus and acrosome at their proximal ends (Fig. 1). The residual body, not stainable with nuclear dyes, remained connected with the sperm tail when released into the tubular lumen.

Ultrastructural study of the testicular tissue from the biopsy demonstrated an orderly organization of the germinal epithelium up to the beginning of spermatid differentiation, which, in the normally developing human testis, can be divided into eight steps relating to acrosome formation, nuclear condensation and development of the tail (Holstein, 1976; Holstein \& Roosen-Runge, 1981). In the present material, differentiation started in the normal way during steps 1 and 2 . An inconspicuous cytocentrum gave rise to an acrosomal vesicle and a nearby flagellar anlage consisting of two centrioles in $\mathrm{T}$-formation with a protruding axoneme from the distal one. After having moved apart, the acrosomal vesicle and flagellar anlage reached their positions at opposite sides of the nucleus. During further differentiation the normal course of acrosome formation and of nuclear condensation was observed. Also, the flagellar anlage at first became correctly implanted at the nucleus. The basal plate, apposed to the outer nuclear membrane in the implantation fossa, was in contact with the proximal centriole. Perpendicular to this the distal centriole was found, which again distally contacted the plasmalemma (Fig. 2a). Here the emerging axoneme was surrounded by a typical annulus. This arrangement could be recognized until the beginning of step 3 of differentiation, when the slightly ovoid nucleus began to condense. The first signs of irregularities in the development of the tail were then indicated by a reduced presence or even the absence of the pericentriolar granular material previously present in the cytocentrum. Because of this lack, the striated columns of the neck piece were not formed. Simultaneously the distal centriole with the protruding axoneme began to dissociate from the proximal centriole. The correct $T$-formation was no longer maintained (Figs $2 b, c)$. The head and the tail of the spermatozoon subsequently developed independently from each other. The proximal centriole from which normally a centriolar adjunct emerged remained connected with the nucleus (Figs $2 b, 2 c \& 3$ ). The principal piece and the end piece of the tail differentiated normally, and further malformations were particularly associated with the middle piece. The spindle-shaped body often failed to appear or was malformed (Fig. 4b). Consequently the mitochondria were not always arranged as usual (Fig. 4a) around the axoneme, and the annulus did not reach its final position at the transition from the middle to the principal piece (Figs $4 \mathrm{~b}-\mathrm{d}$ ). Furthermore, because of the missing neck piece with its striated columns, a transformation of the distal centriole did not take place and it was often still recognizable throughout the length of the mature tail. The consecutive steps of dissociation of the centrioles

Fig. 1. Light microscopic aspect of a smear of decapitated spermatozoa from an ejaculate. Many of the sperm tails proximally show a knob-like cytoplasmic thickening (arrows) instead of a normal sperm head. $\times 1700$.

Fig. 2. Stepwise dissociation of the distal and proximal centrioles during early spermatid differentiation. (a) After regular implantation at the distal end of the nucleus both centrioles are still found in T-formation. $\times 25200$. (b, c) Early dissociation of the distal centriole, while the proximal one remains attached to the nucleus. The striated columns of the neck piece have failed to appear, and the T-formation cannot be maintained. bp, basal plate; dc, distal centriole; lb, lateral junctional body; pc, proximal centriole. $\times 25600$. 

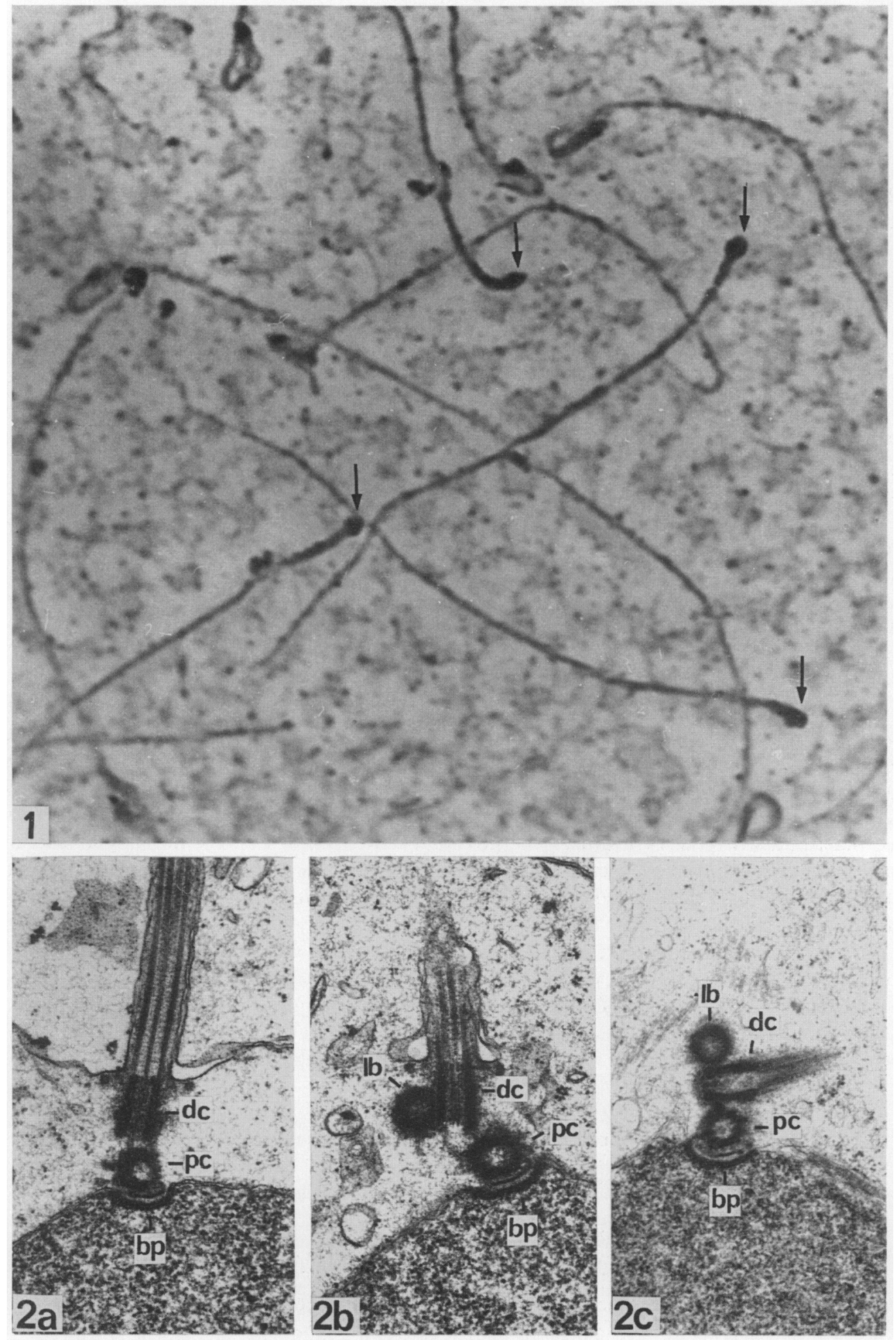


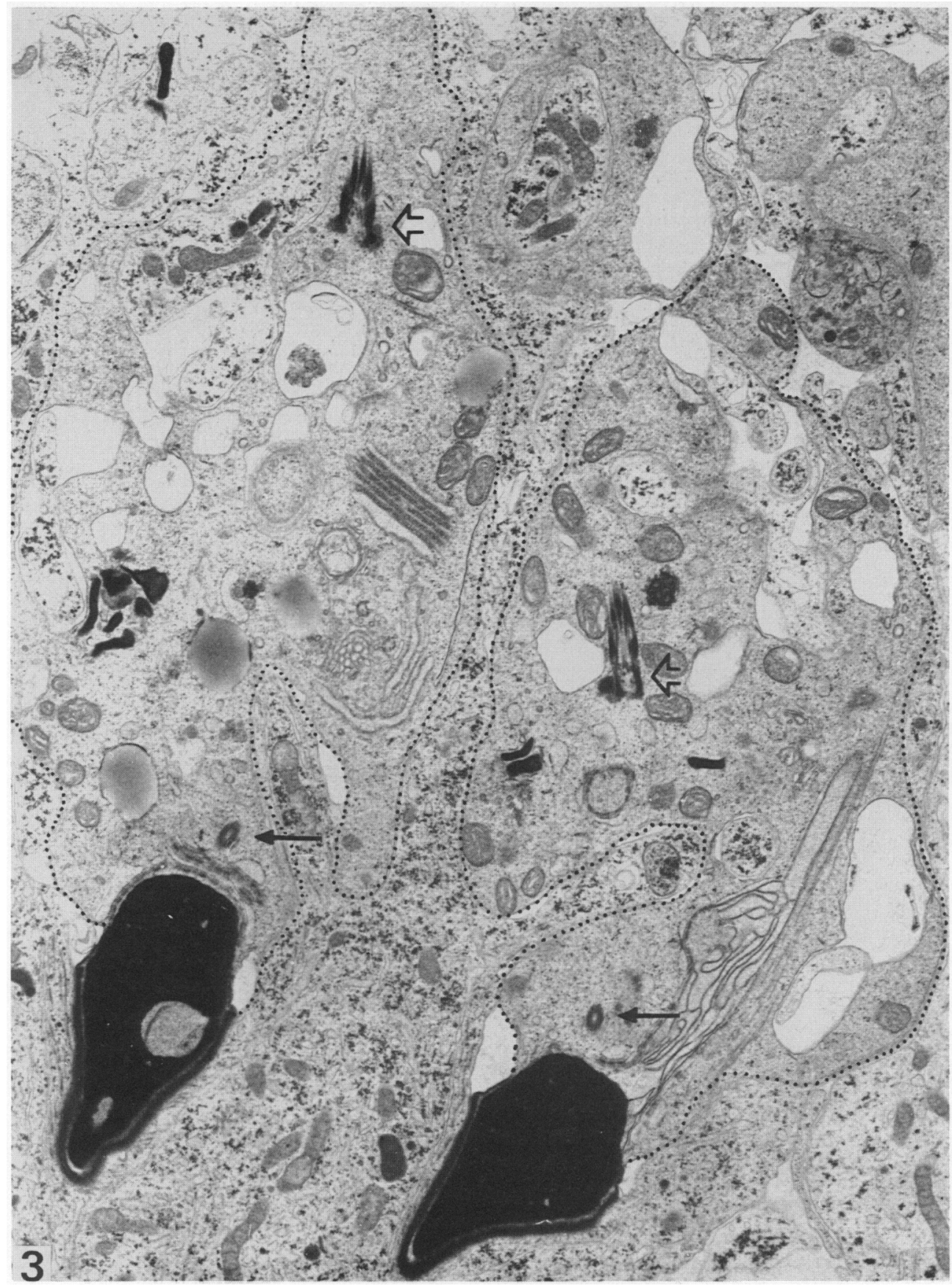

Fig. 3. Late spermatids after final dissociation of the centrioles. $\downarrow$ proximal centriole; $\Downarrow$ distal centriole with outgrowing tail; dotted lines $=$ outlines of the whole spermatids. $\times 11800$.

Fig. 4. Different possibilities of tail development after dissociation of the distal centriole. (a) All the substructures of the tail, arising from the distal centriole, are normally developed. The cytoplasmic droplet remains connected with the proximal end of the flagellum as a knob-like thickening. (b-d) Because of a malformed (b) or missing (c, d) spindle-shaped body, the middle piece of the tail is malformed; neither the mitochondria nor the annulus have reached their final positions. an, annulus; ri, ribosomes; sb, spindle-shaped body. $\times 15000$. 

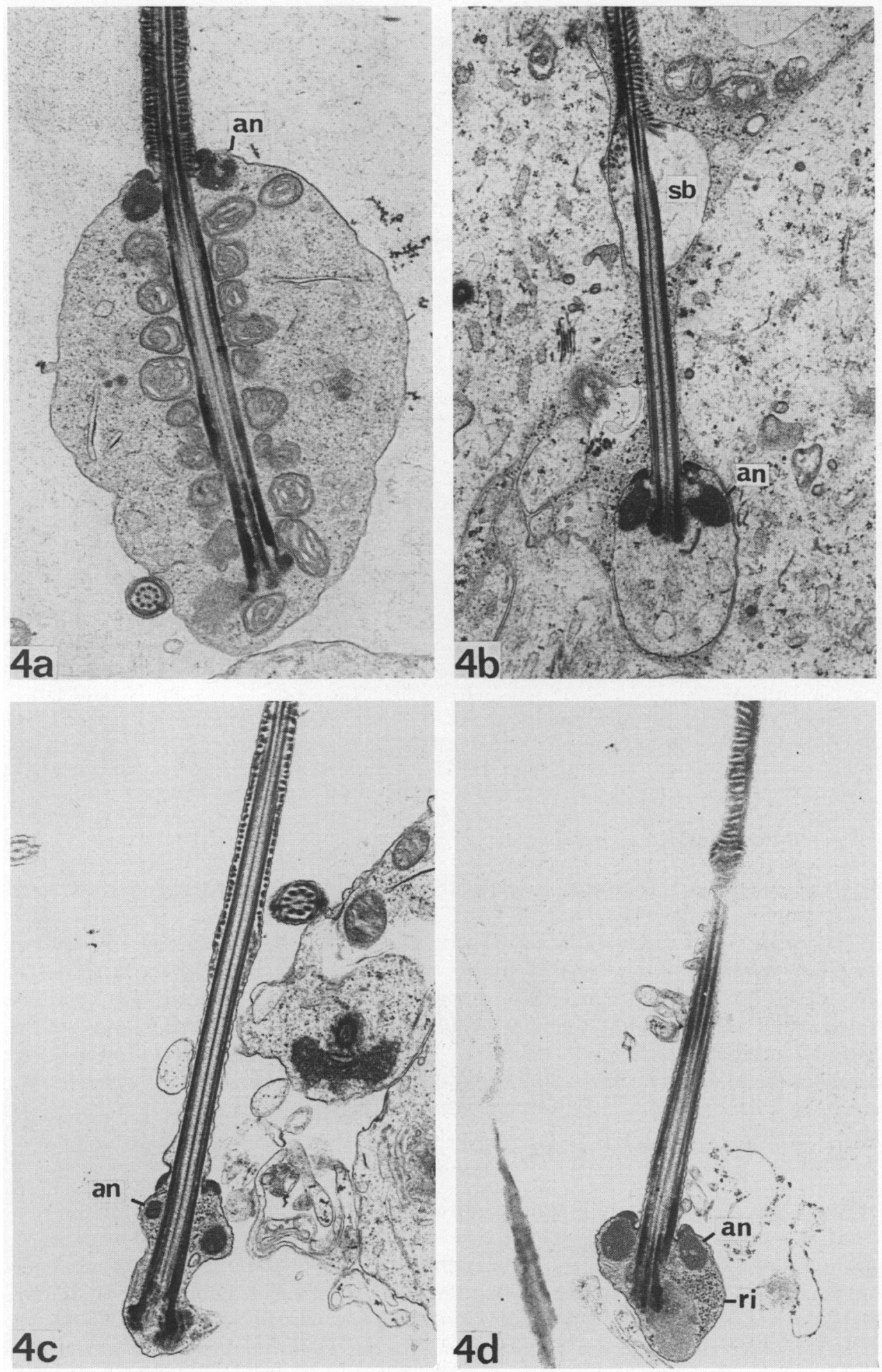


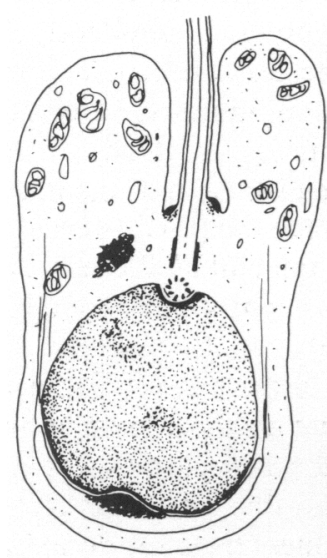

a

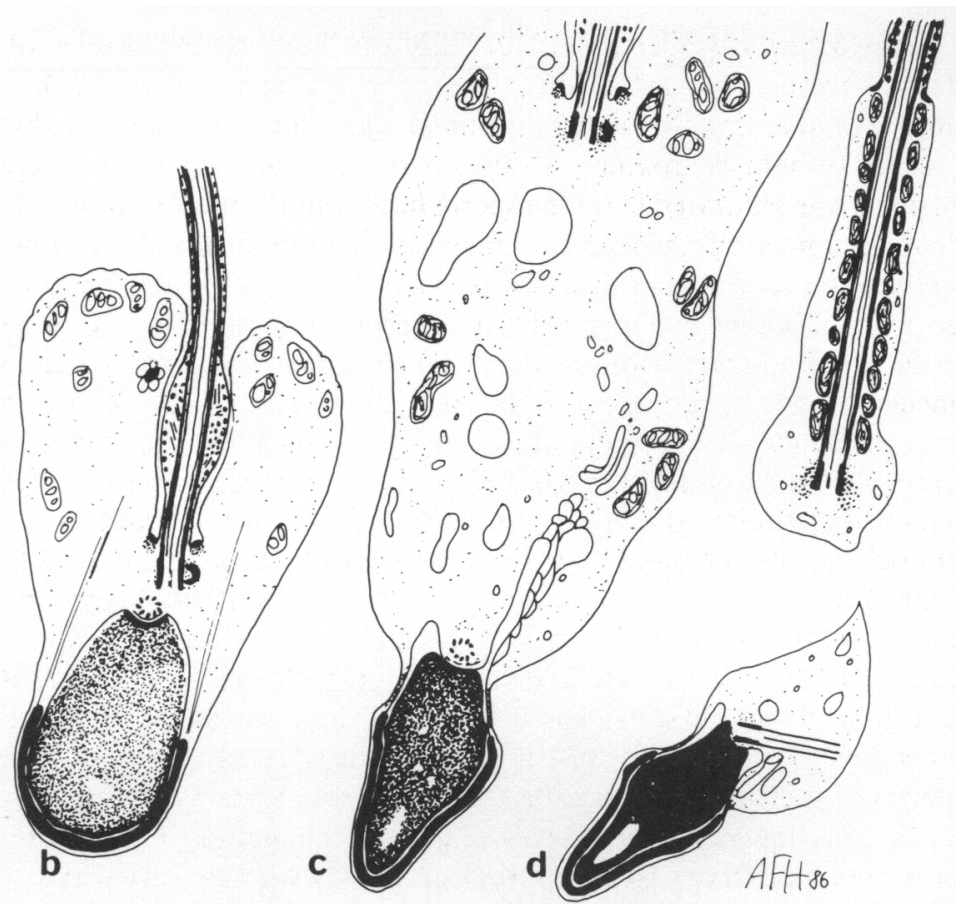

5

Fig. 5. Semi-schematic drawings, based on electron micrographs, of single steps of dissociation of the centrioles during spermatid differentiation. (a) The centrioles are still in T-formation. (b) The T-formation of the centrioles is dissolved. The proximal centriole is attached to the spermatid nucleus, the distal one has developed the flagellar structures. (c) Nucleus and flagellar anlage are found far from each other. (d) The connection between flagellum and nucleus of the spermatid is interrupted. The proximal centriole, which has formed a centriolar adjunct, is implanted at the nucleus. The distal centriole is found at the proximal end of the flagellum whose further substructures may be quite normally developed.

are summarized in Fig. 5. It represents one of the possibilities of differentiation of the separated tail, corresponding to that of Fig. 4(a).

At the stage of final maturation of the sperm components, the dissociated tails were mostly released into the lumina of the seminiferous tubules (Figs $4 a, c, d$ ), while the sperm heads, bearing the proximal centrioles, often remained in the germinal epithelium or sometimes were released separately. After delivery from the germinal epithelium the sperm tails lacking heads are the predominating constituents of the tubular lumina. The detached tails frequently showed a globular cytoplasmic mass at their proximal ends with the constituents of a residual body, which surrounded the distal centriole (Figs $4 \mathrm{a}, \mathrm{c}, \mathrm{d}$ ). Separate residual bodies were rarely found.

\section{Discussion}

In the study reported here a hitherto unknown malformation of human spermatids has been described. It was manifest as a high percentage of decapitated spermatozoa and was caused by a dissociation between the proximal and distal centrioles during the first steps of spermatid differentiation. Because of the centriolar dissociation the sperm head and tail developed separately, and finally a large number of isolated tails was found in the ejaculate. This so-called 'decapitated sperm defect', when observed as a general phenomenon, is a rare malformation. Up to now it has been described ultrastructurally in man by Perotti et al. (1981) and Baccetti et al. (1984), and in the bull 
by Blom \& Birch-Andersen (1970). Electron microscopic studies of the morphogenesis of this defect, however, revealed a different history of origin in each particular case although the result, namely the quantity of isolated tails in the ejaculate, seemed to be always the same when observed by means of light microscopy. In the previous reports the total flagellar anlage consisting of both centrioles was separated from the sperm head, but the malformation described in this study showed a dissociation within the flagellar anlage so that the proximal centriole always remained in connection with the sperm head. This defect gives information about a series of cytological details and their mutual influence. Obviously, the sperm tail is capable of differentiating independently from the nucleus and even without intact $T$-formation of the centrioles. The distal centriole alone is sufficient for the development of the complete flagellum and its autonomous motility, observed for up to nearly $40 \%$ of 'spermatozoa' in the ejaculate. The proximal centriole, on the other hand, induces the formation of the basal plate in the implantation fossa at the distal end of the nucleus. An incorrect implantation fossa, however, inhibits normal contact between the proximal centriole and nucleus, and the basal plate becomes separate, as demonstrated for the bull by Blom \& BirchAndersen (1970). In the present case this contact, although sometimes slightly shifted along the nuclear membrane, was always normal, and the loosening occurred between the centrioles. This means that only the intact $\mathrm{T}$-formation of both centrioles guarantees the correct connection between head and tail of the spermatozoon. The $T$-formation itself presumably will be fixed by the connecting piece, which arises in the form of striated columns from the pericentriolar granular material of the cytocentrum. The defect reported here may be due to the absence of this material with the consequence of non-developing striated columns. It therefore differs from the decapitated sperm defect observed by Baccetti et al. (1984) who suggested that an overproduction of membranes and vesicles by the Golgi complex was responsible for the separation between sperm head and flagellar anlage. All the cytoplasmic components of the developing spermatid are obviously under the influence of the distal centriole and are associated with the isolated tails when released into the tubular lumen. This fact explains the frequent appearance of a cytoplasmic knob containing the constituents of a residual body (Breucker et al., 1985) at the proximal end of the separated tail. The varied aspects of the isolated tails in the lumina of the seminiferous tubules are due to different degrees of development of the middle piece. Perotti et al. (1981), in their study of decapitated spermatozoa in an infertile man, found similarly different types of isolated tails in the ejaculate, although this decapitation defect resulted from a separation of the centrioles as a whole. In both cases the mechanisms responsible for these variations are unknown.

The morphological analysis of the present malformation offers a reliable, although negative, prognosis for the patient. It is, moreover, of special importance for the knowledge of the cytological interrelations between different cell components during human spermatid differentiation.

We thank Mrs Elke Schäfer for excellent technical assistance. Financial support was provided by the Deutsche Forschungsgemeinschaft.

\section{References}

Baccetti, B., Selmi, M.G. \& Soldani, P. (1984) Morphogenesis of 'decapitated' spermatozoa in a man. $J$. Reprod. Fert. 70, 395-397.

Blom, E. \& Birch-Andersen, A. (1970) Ultrastructure of the 'decapitated sperm defect' in Guernsey bulls. $J$. Reprod. Fert. 23, 67-72.

Breucker, H., Schäfer, E. \& Holstein, A.F. (1985) Morphogenesis and fate of the residual body in human spermiogenesis. Cell Tissue Res. 240, 303-309.

Faidt, W. (1979) Elektronenmikroskopie fehlgebildeter Spermatozoen: Literaturübersicht und Mitteilung von zwei eigenen Fällen. Dissertation Universität München.
Holstein, A.F. (1975) Morphologische Studien an abnormen Spermatiden und Spermatozoen des Menschen. Virchows Arch. (Pathol. Anat.) 367, 93-112.

Holstein, A.F. (1976) Ultrastructural observations on the differentiation of spermatids in man. Andrologia 8, $157-165$.

Holstein, A.F. \& Roosen-Runge, E.C. (1981) Atlas of Human Spermatogenesis. Grosse, Berlin.

Perotti, M.E., Giarola, A. \& Gioria, M. (1981) Ultrastructural study of the decapitated sperm defect in an infertile man. J. Reprod. Fert. 63, 543-549. 